\title{
Article \\ Parasitic Coupling in 3D Sequential Integration: The Example of a Two-Layer 3D Pixel ${ }^{\dagger}$
}

\author{
Petros Sideris ${ }^{1,2} \mathbb{E}$, Arnaud Peizerat ${ }^{2}$, Perrine Batude ${ }^{2}$, Gilles Sicard ${ }^{2}$ and Christoforos Theodorou $1, * \mathbb{D}$ \\ 1 University Grenoble Alpes, Univ. Savoie Mont Blanc, Centre National de la Recherche Scientifique (CNRS), \\ Grenoble INstitut Polytechnique (Grenoble INP), Institut de Microélectronique Electromagnétisme \\ Photonique-LAboratoire d'Hyperfréquences et de Caractérisation (IMEP_LAHC), 38000 Grenoble, France; \\ psiderisd@gmail.com \\ 2 Commissariat à l'Energie Atomique et aux Energies Alternatives-Laboratoire d'Electronique des \\ Technologies de l'Information (CEA-LETI), University Grenoble Alpes, 38054 Grenoble, France; \\ arnaud.peizerat@cea.fr (A.P.); perrine.batude@cea.fr (P.B.); gilles.sicard@cea.fr (G.S.) \\ * Correspondence: christoforos.theodorou@grenoble-inp.fr \\ + This paper is an extended version of our paper published in 10th International Conference on Modern \\ Circuits and Systems Technologies (MOCAST), Thessaloniki, Greece, 5-7 July 2021.
}

Citation: Sideris, P.; Peizerat, A.; Batude, P.; Sicard, G.; Theodorou, C Parasitic Coupling in 3D Sequential Integration: The Example of a Two-Layer 3D Pixel. Technologies 2022, 10, 38. https://doi.org/ $10.3390 /$ technologies10020038 Academic Editors: Spiros Nikolaidis and Rodrigo Picos

Received: 11 January 2022

Accepted: 15 February 2022

Published: 28 February 2022

Publisher's Note: MDPI stays neutral with regard to jurisdictional claims in published maps and institutional affiliations.

Copyright: (C) 2022 by the authors. Licensee MDPI, Basel, Switzerland. This article is an open access article distributed under the terms and conditions of the Creative Commons Attribution (CC BY) license (https:// creativecommons.org/licenses/by/ $4.0 /)$.

\begin{abstract}
In this paper, we present a thorough analysis of parasitic coupling effects between different electrodes for a 3D Sequential Integration circuit example comprising stacked devices. More specifically, this study is performed for a Back-Side Illuminated, 4T-APS, 3D Sequential Integration pixel with both its photodiode and Transfer Gate at the bottom tier and the other parts of the circuit on the top tier. The effects of voltage bias and 3D inter-tier contacts are studied by using TCAD simulations. Coupling-induced electrical parameter variations are compared against variations due to temperature change, revealing that these two effects can cause similar levels of readout error for the top-tier readout circuit. On the bright side, we also demonstrate that in the case of a rolling shutter pixel readout, the coupling effect becomes nearly negligible. Therefore, we estimate that the presence of an inter-tier ground plane, normally used for electrical isolation, is not strictly mandatory for Monolithic 3D pixels.
\end{abstract}

Keywords: image sensors; 3D pixels; 3D sequential integration; 3DSI; monolithic 3D; M3D; coupling; parasitic capacitances

\section{Introduction}

User-interactive applications are continuously emerging and driving the electronics industry towards the adoption of heterogeneous technologies in the sense that the analog sensing parts are integrated together with digital processing parts. The More-than-Moore technology development direction is a key enabler for such heterogeneous integrations, as it involves a wide variety of people-environment interaction applications [1].

An important driving application within the More-than-Moore scheme is the CMOS Image Sensor (CIS), because it is a circuit that requires the heterogeneous integration of different system parts: a photon-to-electron converter (photodiode) functions as the sensing interface in the pixel array and the readout part consists of an analog circuit that transmits information from the pixel to a digital circuit for processing. Moreover, CIS is an ideal candidate for studying coupling effects in 3D integration technologies, as it is highly sensitive to noise (dynamic) and mismatch (static) variations, while simultaneously being an extremely attractive application of 3D Integration for the semiconductor industry [2]. In particular, 3D CIS allows the development of smarter, more advanced sensors by cointegrating different blocks (Analog, Digital and RF) in multiple stacked tiers. By using the 3D stack integration process, the CMOS processing part can be stacked on top of the pixel, enabling the use of more advanced technology nodes for the processing circuit. 
Although, until today, 3D stacking is predominantly used for 3D CIS, constraints concerning the alignment capabilities cannot allow more aggressive pixel miniaturization, which is required for future CIS generations [3,4]. However, in the case of 3D Sequential Integration (3DSI) [5,6], where one tier is processed on top of the other instead of being stacked, this drawback can be overcome, achieving pixel partitioning with state-of-the-art pixel pitch. In addition, 3DSI offers 3D contacts of outstanding high-density between tiers (up to $10^{8} 3 \mathrm{D}$ via $/ \mathrm{mm}^{2}$ ), enabling partitioning with high connectivity and low latency. Therefore 3DSI allows the co-integration of dense logic and memory layers but also heterogeneous components such as MEMS/NEMS for the compact coexistence of sensing and computing [7].

Despite the fact that the 3DSI technology approach offers great opportunities for the domain of CSI, it is also prone to many challenges, such as the limited Thermal Budget (TB) for the fabrication of the top-tier devices. To date, Low Temperature (LT) devices have been successfully fabricated and optimized for both low-voltage (LV) [5] and high-voltage (HV) [8] applications. Using such LT devices, Coudrain et al. [9] have investigated the feasibility of a 3DSI Back-Side Illuminated (BSI) CIS with miniaturized pixels, achieving a photodiode area increase by $44 \%$ for a $1.4 \mu \mathrm{m}$ pitch. However, the other major challenge, concerning the impact of the inter-tier parasitic coupling on a 3DSI CIS performance, has not been examined in depth.

In this work, we present a thorough analysis of the possible coupling effects in the realization of a BSI 4-Transistor (4T) pixel with its diode and Transfer Gate (TG) on the bottom tier and the rest of its circuitry on the top tier of a 3DSI process, as an example of parasitic coupling analysis in a 3DSI circuit. In Section 2, the general principles and operation scheme of a CMOS imager are presented, including a presentation of the basic CIS architectures and the most critical performance metrics. In Section 3, the inter-tier coupling effects are analyzed, first at a single device level and then at the pixel level.

\section{CMOS Imager: Architectures and Integration}

\subsection{CIS Standard Architectures and Operation}

The most basic pixel architecture, called Passive Pixel Sensor (PPS) [10], contains passive pixels with no amplification, with only a photodiode for light detection and transistor switch for row selection, as shown in Figure 1a. Due to the lack of amplification or more sophisticated circuit, this architecture suffers from poor image quality, high KTC noise level and slow readout $[11,12]$.

A major improvement to PPS architecture is the so-called Active Pixel Sensor (APS) [13], which incorporates an in-pixel amplifier for every pixel. Therefore, each pixel is composed of a photodiode (PD), a reset transistor (RST) and a source-follower (SF) amplifier, as demonstrated in Figure 1b. This architecture has reduced power consumption, random access and high-speed readout, thanks to the fact that the readout output is a voltage instead of charge transfer. On the downside, having additional transistors per pixel degrades the Fill-Factor. Finally, the issue of the kTC noise generated by the photodiode reset is not resolved.

In order to address the high reset noise issue, a Pinned Photodiode (PPD) pixel was introduced [14], resulting in the architecture shown in Figure 1c, which is the same as the APS one, but with the PPD connected to the readout circuit. This is achieved by an extra Transfer Gate (TX) and a Sense Node (SN). This 4T-APS architecture further allows the implementation of a fast Correlated Double Sampling (CDS) technique at the column level. Finally, thanks to the superior noise performance of the PPD [3], 4T-APS is currently the preferred architecture for CIS pixels in a variety of applications such as mobile imaging, digital still and video cameras, as well as surveillance cameras.

A schematic representation of the voltage output for a 3T-APS pixel is presented in Figure 2, showing how the operation sequence consists of three stages:

1. Reset (RST ON): The photodiode voltage is set to a reference voltage $\mathrm{V}_{\text {ref; }}$; 
2. Exposure (RST OFF): The detected photons decrease the reverse photodiode voltage during the integration time $\left(\mathrm{t}_{\text {int }}\right)$;

3. Readout (RS and SF ON): The output voltage level is sampled and further processed at the column level.
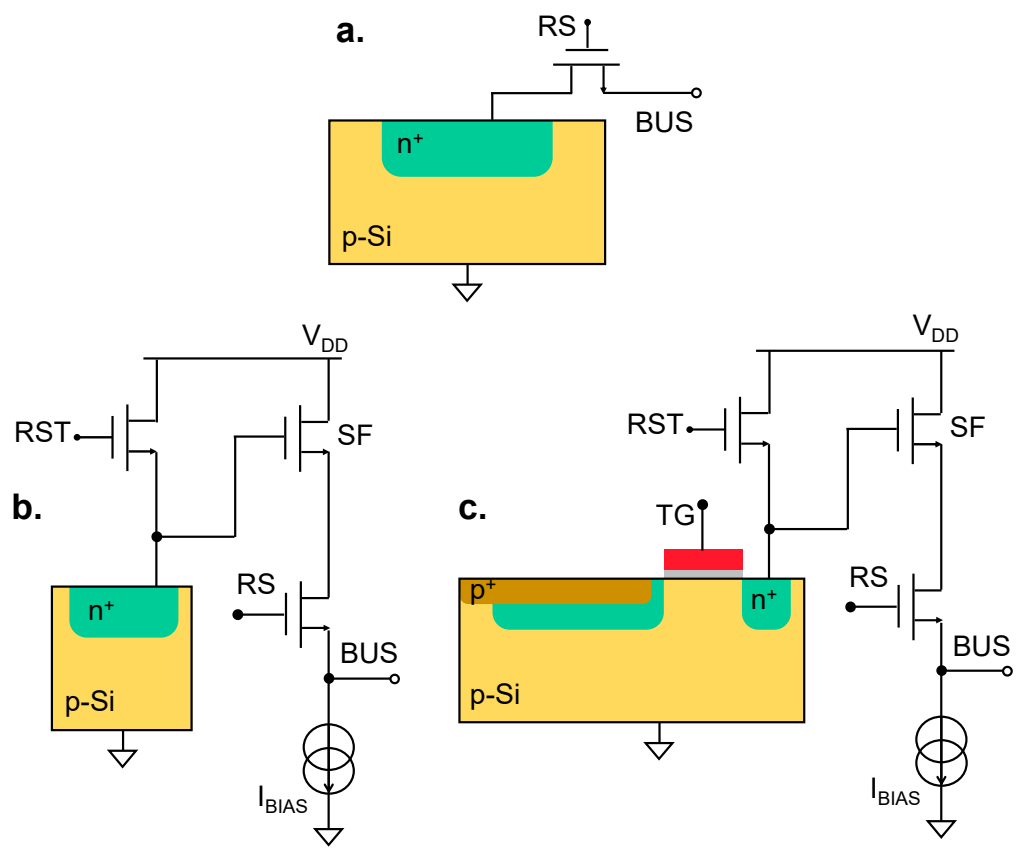

Figure 1. (a) Passive CMOS pixel based on a single in-pixel transistor, (b) active CMOS pixel based on an in-pixel amplifier and (c) active CMOS pixel based on an in-pixel amplifier in combination with a pinned photodiode.

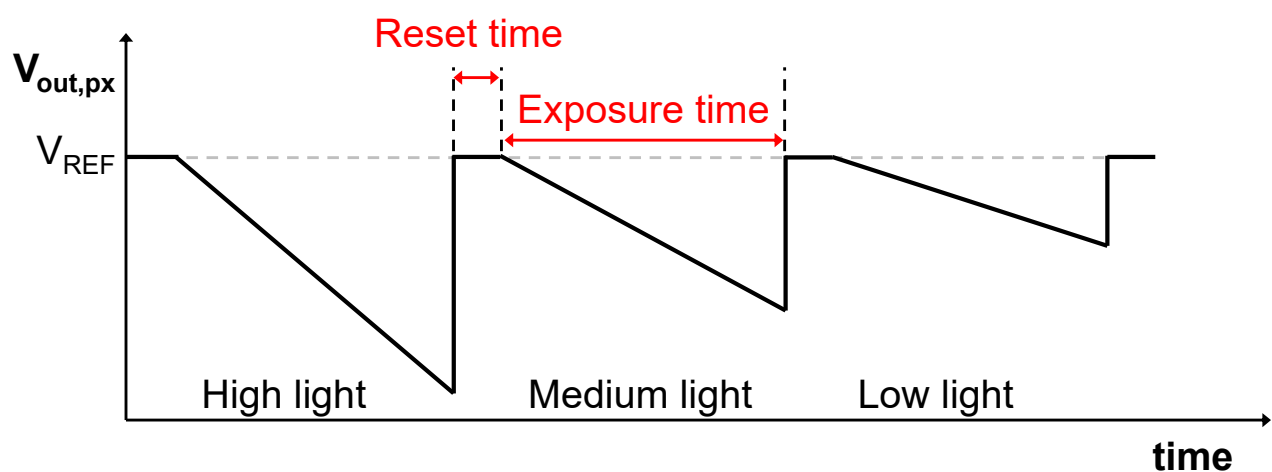

Figure 2. Operation principle of a 3T-APS pixel.

\subsection{The Back-Side Illumination Integration Scheme}

In the case of Front-Side Illumination, the optical path includes the total Back End Of Line (BEOL) thickness, inducing losses and crosstalk between pixels, because of the reflection on the metal lines. By flipping the sensor so that light drops directly on the photodiode without passing through the pixel's BEOL, the BSI integration scheme can be achieved. This is illustrated schematically in Figure 3, along with the example of stacked tiers of a 3D integration. The BSI scheme has become the preference for high-end consumer applications, dictated by the mobile phone market which requires continuously higher resolution for the same sensor size [3,4]. Currently, more than half of the mobile phone market utilize BSI integration [15]. Since 2010, the industrial trend is to combine BSI and 3D stacking/integration to reach ultimate performance, while at the same time maintaining a small pixel size. 


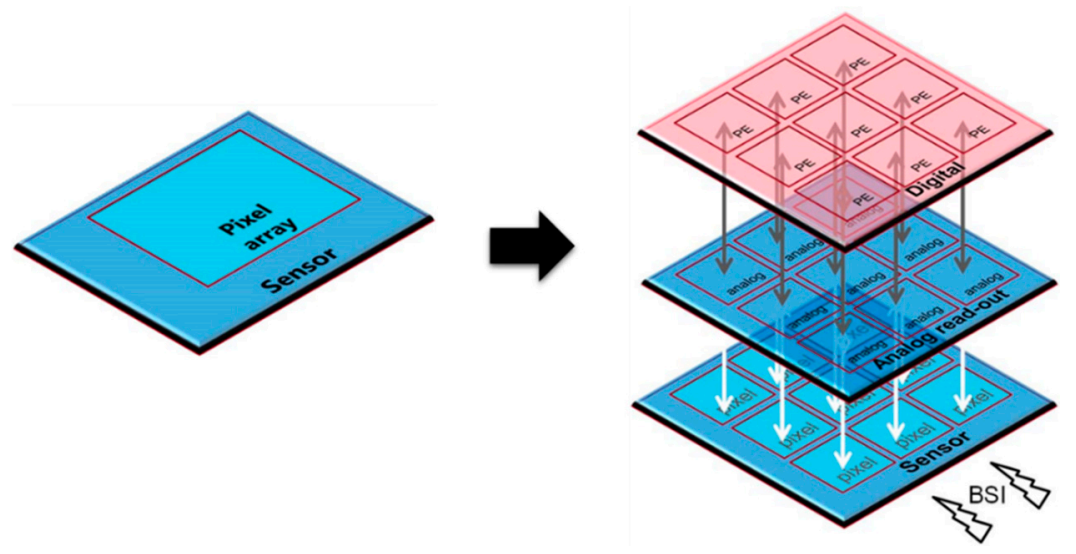

Figure 3. Realization of a CIS with planar (left) and three-dimensional partitioning (right).

\subsection{CIS Performance Metrics}

There are a series of important performance metrics for a CIS that are worth mentioning, before presenting our simulation study, such as the Signal-to-Noise Ratio (SNR), the Dynamic Range (DR) and the Conversion Gain (CG) amongst others.

SNR is defined as the ratio of the useful signal amplitude to the amplitude of undesirable noise and is a good indicator of image quality. Therefore it has to be maximized either by increasing the sensitivity and CG or by decreasing the noise floor $[11,16]$. SNR is reduced in the case of a smaller pixel area, which means less absorbed photons for the same duration. However, the reduction in pixel size provides higher spatial resolution, at a lower light sensitivity as a trade-off. Minimizing the pixel size will also affect the Full Well Capacity (FWC) and, thus, the DR (these parameters will be below).

The Fill Factor (FF) is another parameter that is affected by pixel scaling [11,16]. It is defined as the percentage of the photosensitive area with regards to the total pixel area. Thus, it expresses what portion of the total pixel area is utilized for photon collection. A high FF value means higher sensitivity; thus, it ought to be maximized, for example, by downscaling pixel transistors or by adding microlenses to guide the light towards the photodiode area.

Regarding the sensitivity of a sensor with linear response, as is the case of 3T/4T-APS, it is equal to the slope of the transfer function (in $\mathrm{V} /$ lux.s or $\mathrm{e}^{-} /$lux.s). It corresponds to the change in output potential for a given light intensity and integration time. It is highly dependent on the Quantum efficiency (QE) of the sensor [11], a quantity that shows how efficiently the photons are collected and converted to electrons. One method to maximize it is to use anti-reflecting coating or optimize the stack between the sensor surface and the photodiode, or to use BSI, in order to avoid reflections at the interfaces.

The DR of a CIS is defined as the range of light intensity that can be measured with no distortion by the sensor $[11,16]$. It can be calculated as $D R=20 \log \left(S_{\max } / S_{\min }\right)$, where $S_{\max }$ is the highest detectable signal and $S_{\min }$ the lowest one (essentially the noise floor). On the other hand, $S_{\max }$ is limited by the Full Well Capacity and pixel saturation.

An important parameter for our study is CG, which is defined as $C G=\Delta V_{\text {out }} / N_{e}$, where $\Delta V_{\text {out }}$ is the pixel output $\left(\mathrm{V}_{\text {out,int }}-\mathrm{V}_{\text {out,ref }}\right)$ when the number of electrons is equal to $\mathrm{N}_{\mathrm{e}}$ in a single packet $[11,16] . \mathrm{N}_{\mathrm{e}}$ depends on photon flux and QE. It is measured in $\mathrm{V} / \mathrm{e}^{-}$ and it characterizes the charge-to-voltage conversion; thus, a high CG results in higher sensitivity, especially at low light. Another method to express CG is through (X), taking into account $\mathrm{SN}$ capacitance, $\mathrm{C}_{\mathrm{SN}}$, and any additional parasitic capacitance, $\mathrm{C}_{\mathrm{P}}$.

$$
\mathrm{CG}=\frac{q}{\mathrm{C}_{\mathrm{SN}}+\mathrm{C}_{\mathrm{P}}}
$$

Finally, the amount of charge that can be detected without reaching saturation is expressed through the FWC metric, which is measured in number of charges and determines 
the sensor's DR [11,16]. If we neglect the noise, FWC can be roughly approximated by $\mathrm{FWC}=\mathrm{qC}_{\mathrm{PD}} \mathrm{V}_{\mathrm{PD}}$, where $\mathrm{V}_{\mathrm{PD}}$ is the applied voltage across the photodiode, and $\mathrm{C}_{\mathrm{PD}}$ is the photodiode capacitance. By increasing $\mathrm{C}_{\mathrm{PD}}$, therefore, one can directly increase the sensor's FWC. However, in that case, CG would be decreased due to the increased capacitance. This in turn may result in a range decrease at the low intensity end, and this contradiction is actually the well-known DR/sensitivity tradeoff.

\section{Parasitic Capacitance Coupling in a Two-Layer 3DSI Pixel}

As already mentioned in the Introduction, pixel partitioning in two layers of a 3DSI process is a very promising technique to boost an imager's performance by increasing the photodiode area ratio, and it has dedicated layers for each type of circuit (read-out, digital etc.). Nevertheless, positioning transistors right above the photodiode's transfer gate at a submicrometre distance introduces a high risk of parasitic capacitance coupling, which will be investigated in this Section. At first, in order to be certain if there can be a significant coupling-induced threshold voltage shift and, if so, to quantify it, we performed simulations at a device level, without taking into account the particularities of a pixel's circuit operation and chronogram. Afterwards, in Section 3.2, we present the simulation results we obtained at a circuit level, examining in which cases the parasitic coupling can affect or not the pixel's output precision.

\subsection{Investigation at Device Level}

\subsubsection{Simulated Structure Details}

In order to carry out our study, the simulation structure depicted in Figure 4 was considered. As shown in the cross-section of Figure 4a, our setup has its pixels sequentially integrated in such a manner that PPD and TG are placed at the bottom layer and the rest of the readout circuitry is placed right above them, with an Inter-Layer Dielectric (ILD) of $200 \mathrm{~nm}$ thickness separating them. TG can toggle between 0 and $\mathrm{V}_{\mathrm{DD}}$, enabling photo-generated electrons to be transferred from the photodiode area to SN and then to the drain of RST and the gate of the SF via a 3D contact.
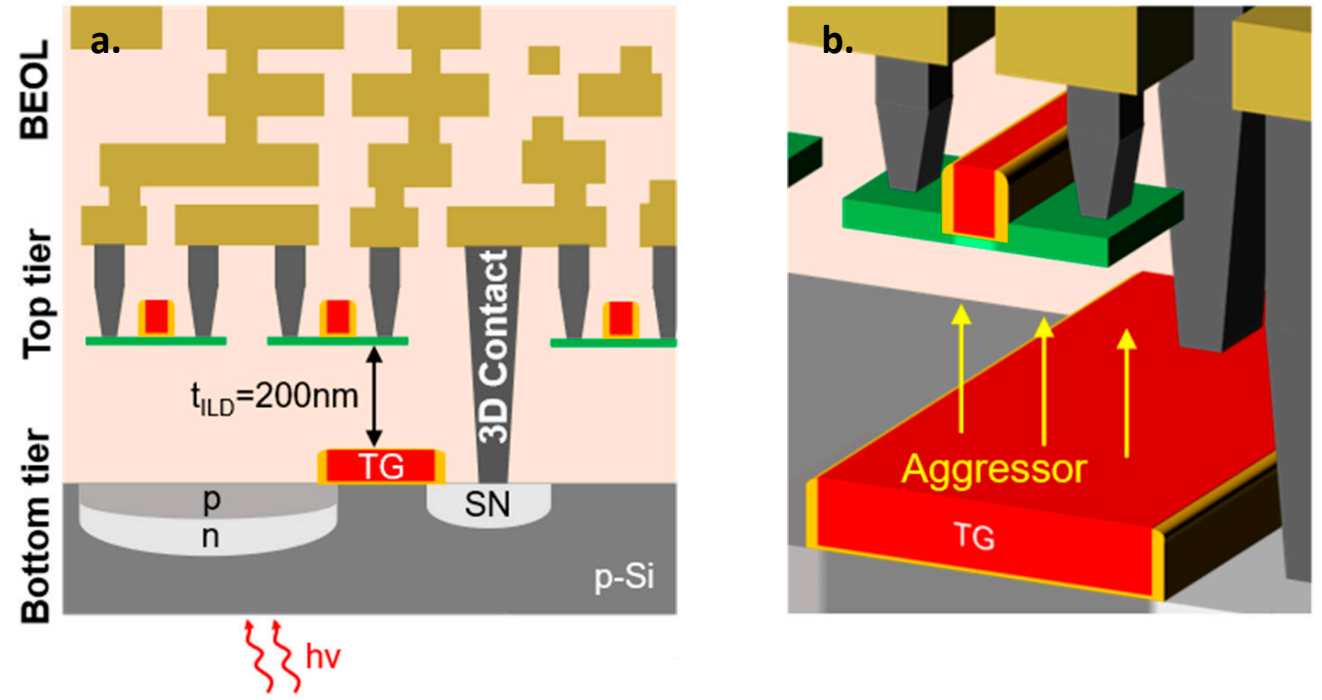

Figure 4. (a) Cross-section of $4 \mathrm{~T}$ pixel, partitioned in 3DSI. (b) In the most critical case, the top device is placed right above the TG electrode carrying a voltage that can go up to $\mathrm{V}_{\mathrm{DD}}=2.5 \mathrm{~V}$.

In order to extend the voltage swing of top-tier devices, analog devices with power supply of $2.5 \mathrm{~V}$ were considered for the top layer. In addition, the most critical condition of inter-tier coupling has been chosen by placing the TG electrode right under each one of the top layer devices, as shown in Figure $4 b$. 


\subsubsection{Impact of Inter-Tier Coupling on Electrical Parameters}

In order to assess whether inter-tier static coupling can be detrimental to the functionality of the 3DSI pixel, we investigated the impact of the capacitive coupling of the TG placed at the bottom tier on each top device performance. By varying the TG gate voltage bias within its normal operation limits $(0-2.5 \mathrm{~V})$, we observe a shift of the $I_{D}-V_{G}$ characteristics for the top devices, as shown in Figure 5a. This behavior can be attributed to the fact that the top devices are actually asymmetrical SOI structures, where ILD plays the role of the Buried Oxide (BOX). It is well-known in SOI devices [17] that this effect is nonlinear, with its maximum in weak inversion and equal to a constant value when the device enters strong inversion.
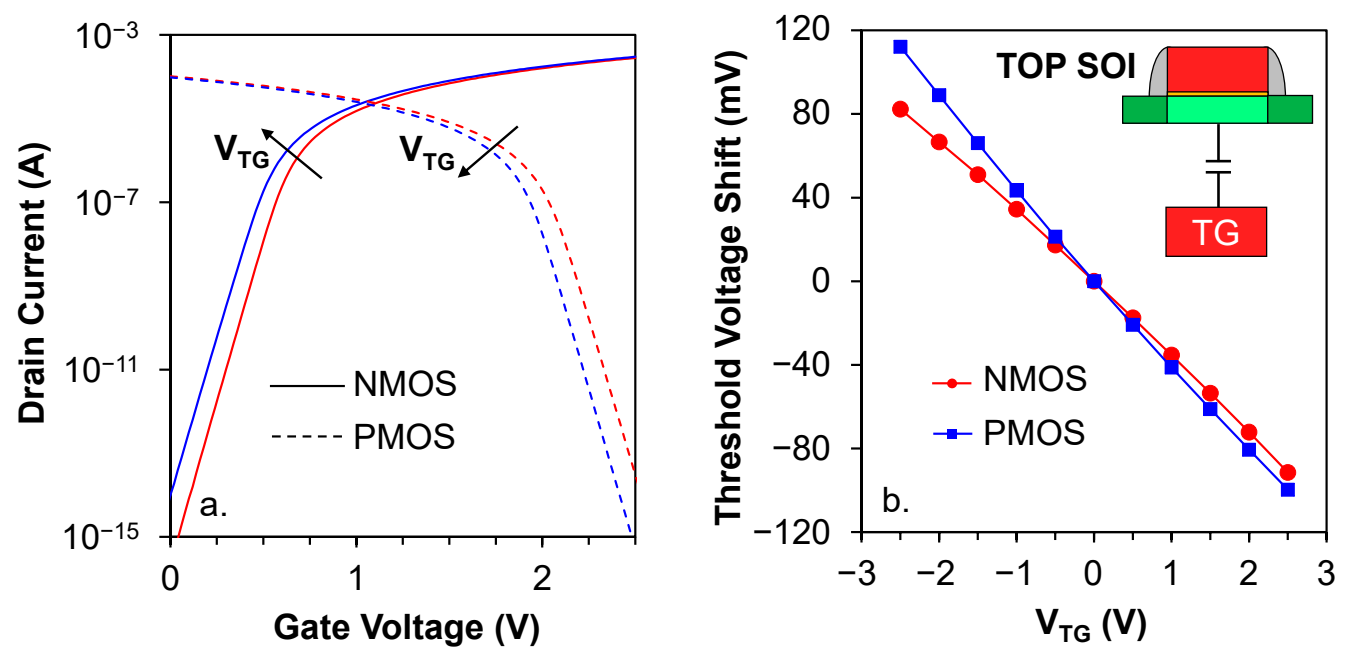

Figure 5. (a) Impact of $\mathrm{TG}$ coupling $\left(\mathrm{V}_{\mathrm{TG}}=0\right.$ (red) $-2.5 \mathrm{~V}$ (blue)) on the input characteristics for NMOS and PMOS devices. (b) Extracted threshold voltage shift versus TG voltage bias ( $-2.5 \mathrm{~V}$ to $2.5 \mathrm{~V}$ with $0.5 \mathrm{~V}$ step).

Figure $5 \mathrm{~b}$ shows the extracted threshold voltage shift $\left(\Delta \mathrm{V}_{\mathrm{TH}}\right)$ versus $\mathrm{V}_{\mathrm{TG}}$ (from $-2.5 \mathrm{~V}$ to $+2.5 \mathrm{~V}$ ) and a nearly linear relation was obtained, which allowed the extraction of the back-bias efficiency $\gamma$ for the top devices ( $35 \mathrm{mV} / \mathrm{V}$ for NMOS and $42 \mathrm{mV} / \mathrm{V}$ for PMOS). $\mathrm{V}_{\mathrm{TH}}$ was extracted using the constant current method [18] for each $\mathrm{I}_{\mathrm{D}}-\mathrm{V}_{\mathrm{G}}$ curve.

In order to evaluate the strength of TG-induced static coupling on the top device performance, we benchmarked it against a temperature variation of $100{ }^{\circ} \mathrm{C}$, which is a typical range in consumer electronics. Contrary to capacitive coupling, the effect of temperature varies depending on the gate voltage $\mathrm{V}_{\mathrm{G}}$. Indeed, our simulations (Figure 6) show that when proceeding from $253 \mathrm{~K}$ to $353 \mathrm{~K}\left(-20^{\circ} \mathrm{C}\right.$ to $\left.80{ }^{\circ} \mathrm{C}\right)$, an increase in the leakage current and a decrease in ON current were observed, whereas at a specific gate voltage around $\mathrm{V}_{\mathrm{TH}}$, the drain current remains unaffected by $\mathrm{T}$. This effect is well known in the literature and is due to the canceling out between the rise in carrier concentration with temperature for low $V_{G}$ and the decrease in carrier mobility at high $V_{G}$ [19]. The voltage at which this happens is characterized as the Zero Temperature Coefficient (ZTC) point [20].

Eventually, the $\mathrm{I}_{\mathrm{D}}-\mathrm{V}_{\mathrm{G}}$ shift resulting from both coupling and temperature variation can be translated in an alteration of the top device electrical parameters, namely the $\mathrm{V}_{\mathrm{TH}}$, the leakage current $\left(\mathrm{I}_{\mathrm{OFF}}\right)$ and the saturation current $\left(\mathrm{I}_{\mathrm{ON}}\right)$. The comparison of the extracted parameters for the two effects is shown in Figures 7 and 8. The results show that they are approximately in the same order of magnitude while the limited $\Delta \mathrm{V}_{\mathrm{TH}}$ due to the temperature variation is attributed to the ZTC point near $\mathrm{V}_{\mathrm{TH}}$. Moreover, as observed in Figure 7 , $\mathrm{I}_{\mathrm{OFF}}$ is significantly shifted with $\mathrm{V}_{\mathrm{TG}}$, which can be detrimental for memory blocks comprising switch transistors, placed at the top-tier above TG. 

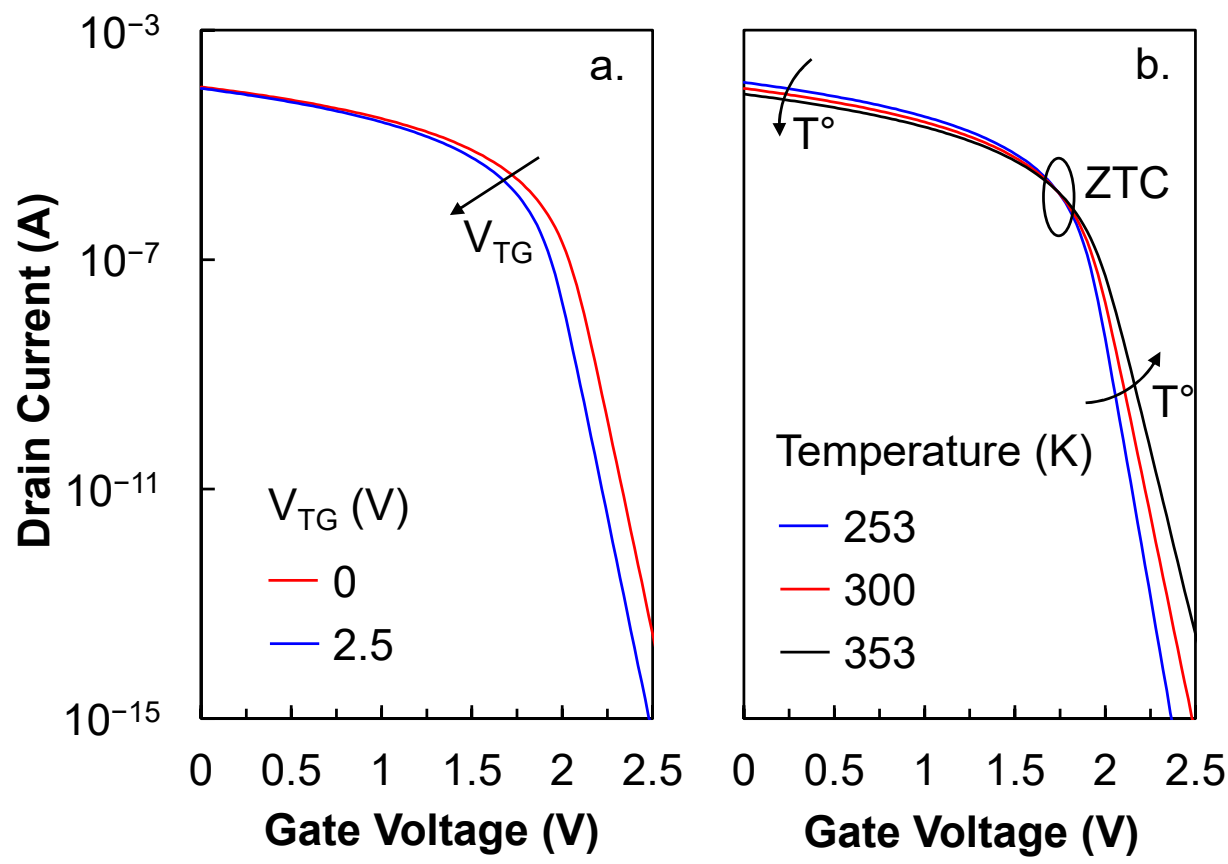

Figure 6. Impact of coupling (a) and temperature variation (b) on the top-tier SOI input characteristics.

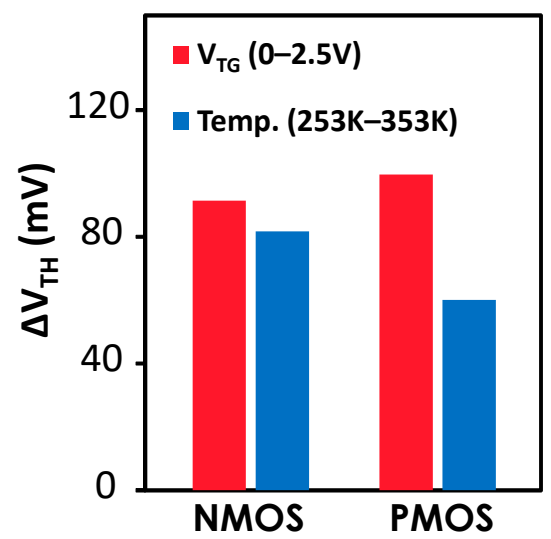

Figure 7. Comparison of the $\mathrm{V}_{\mathrm{TH}}$ variation of the top-tier NMOS and PMOS due to TG coupling or due to a $100 \mathrm{~K}$ temperature increment.
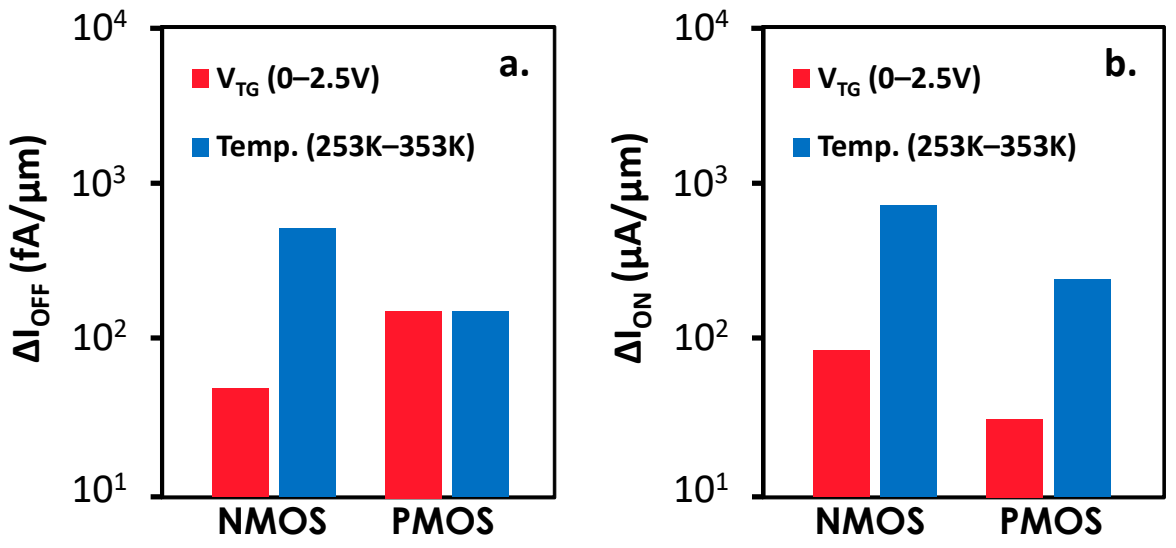

Figure 8. Comparison of the $\mathrm{I}_{\mathrm{OFF}}(\mathbf{a})$ and $\mathrm{I}_{\mathrm{ON}}(\mathbf{b})$ variation of the top-tier NMOS and PMOS due to TG coupling or due to a $100 \mathrm{~K}$ temperature increment. 


\subsection{Investigation at Pixel Level}

\subsubsection{Pixel Topology and Chronogram}

In order to carry out our analysis at the pixel level, the 4T-APS topology was selected, which consisted of an NMOS-TG and the RST, SF and RS in a PMOS circuit configuration, as illustrated in Figure 9a. As shown from the chronogram of the 4T-APS pixel readout operation presented in Figure 9b, during the readout cycle, TG was switched ON following the $\mathrm{SN}$ reset, allowing the diffusion of photo-generated electrons, which in turn cause a voltage drop at the input of the SF. Due to the rough unity gain of SF, the voltage drop is transferred nearly at the same level at its output.
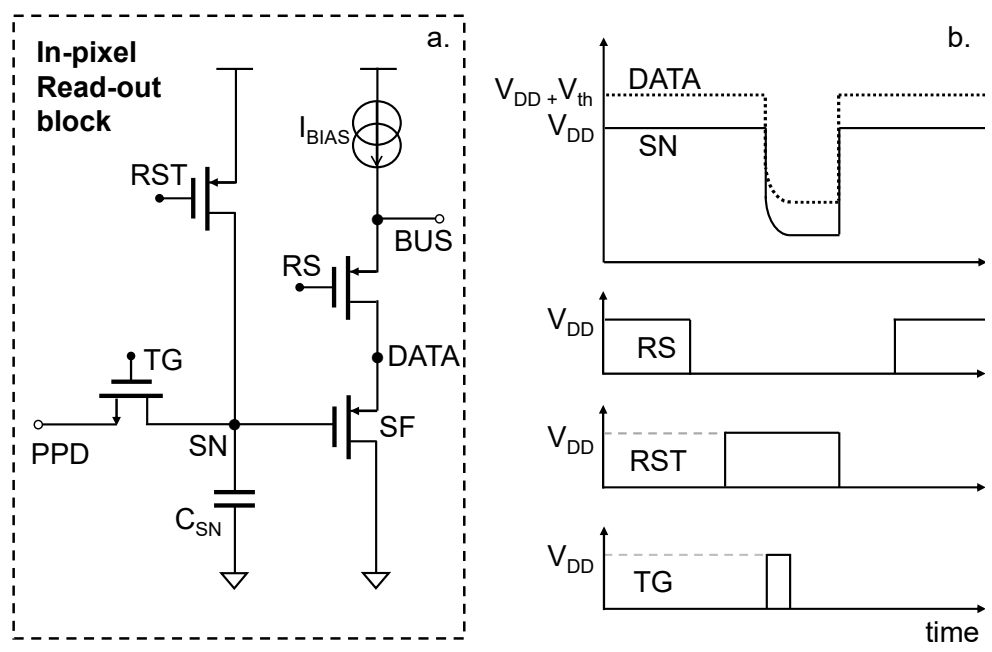

Figure 9. (a) 4T pixel readout circuit. (b) chronogram of a readout cycle.

The efficiency of the aforementioned operation is characterized by the CG in $\mu \mathrm{V} / \mathrm{e}^{-}$, given by the following [21]:

$$
\mathrm{CG}=\frac{q \mathrm{G}_{\mathrm{SF}}}{\mathrm{C}_{\mathrm{SN}}+\mathrm{C}_{\mathrm{GD}}+\left(1-\mathrm{G}_{\mathrm{SF}}\right) \mathrm{C}_{\mathrm{GS}}},
$$

where $q$ is the elementary charge, $\mathrm{G}_{\mathrm{SF}}$ is the $\mathrm{SF}$ gain, $\mathrm{C}_{\mathrm{SN}}$ is the sum of parasitic capacitances at the $\mathrm{SN}$ node and $\mathrm{C}_{\mathrm{GS}}$ and $\mathrm{C}_{\mathrm{GD}}$ are the gate to source and gate to drain capacitances of $\mathrm{SF}$.

The gain $\mathrm{G}_{\mathrm{SF}}$ of the SF, on the other hand, is expressed as follows:

$$
\mathrm{G}_{\mathrm{SF}}=\frac{\mathrm{g}_{\mathrm{m}, \mathrm{SF}}}{\mathrm{g}_{\mathrm{ms}, \mathrm{SF}}}=\frac{1}{\mathrm{n}^{\prime}}
$$

where $\mathrm{g}_{\mathrm{m}, \mathrm{SF}}$ and $\mathrm{g}_{\mathrm{ms}, \mathrm{SF}}$ are the gate and source transconductances, respectively, and $\mathrm{n}$ is the body factor of SF. The gain is approximately equal to unity in the case where the BG of $\mathrm{SF}$ can be tied to the source or else it is process-dependent and is given by $n=1+\gamma$, where $\gamma$ is the back-bias efficiency. With the increase in ILD thickness, $\mathrm{n}$ approaches unity. For the $\gamma$ values extracted in the previous section, we have evaluated the gain $\mathrm{G}_{\mathrm{SF}}$ of the NMOS and PMOS devices as 0.97 and 0.96 , respectively.

The cutoff frequency $\left(\mathrm{f}_{\mathrm{c}}\right)$ of the SF is given by the following [21]:

$$
f_{c}=\frac{g_{m, S F}}{2 \pi \cdot C_{o u t, S F} \cdot G_{S F} \cdot\left(C_{S N}+C_{G D}+C_{G S}\right)},
$$

where $\mathrm{C}_{\text {out,SF }}$ is the capacitance observed at the source of the SF that is the column-level capacitance if there are no other stages in between. 


\subsubsection{Impact of TG Coupling on Pixel Electrical Parameters}

In order to evaluate 3DSI impacts on the two critical parameters CG and $f_{c}$ analyzed above, parasitic extraction was performed concerning a single- and a two-layer implementation of our pixel, as illustrated in Figure 10, and the results along with CG values are presented in Table 1 . As observed, the sums of $\mathrm{C}_{\mathrm{SN}}$ and $\mathrm{C}_{\mathrm{GD}}$, as well as $\mathrm{C}_{\mathrm{GS}}$, slightly increased by $48 \mathrm{aF}$ and $44 \mathrm{aF}$, respectively, which may be attributed to the proximity of the top tier.

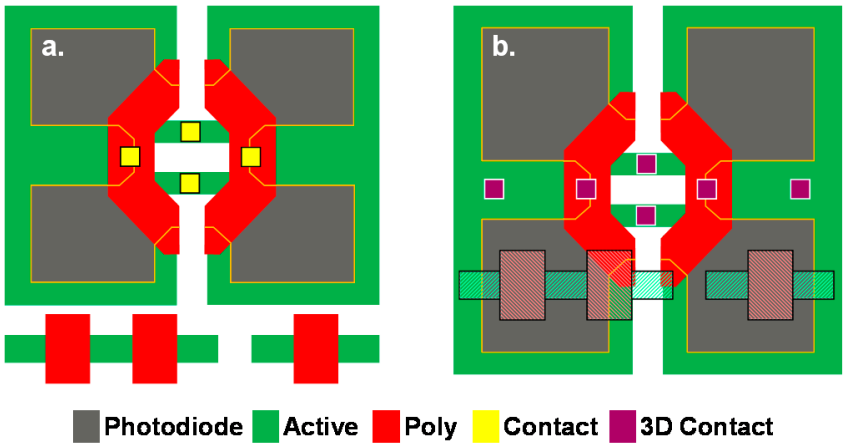

Figure 10. Shared pixels at photodiode layer: 2D (a) vs. 3D (b). Photodiode area (in grey) increased by $44 \%$ when the three readout transistors were placed at the top tier.

Table 1. Two-dimensional vs. three-dimensional parasitic capacitances and CG.

\begin{tabular}{ccccc}
\hline & $\mathrm{C}_{\mathrm{SN}}+\mathrm{C}_{\mathrm{GD}}(\mathbf{f F})$ & $\mathrm{C}_{\mathrm{GS}}(\mathbf{f F})$ & $\mathrm{C}_{\text {out,SF }}(\mathrm{pF})$ & $\mathrm{CG}\left(\mu \mathrm{V} / \mathbf{e}^{-}\right)$ \\
\hline 2D & 4.432 & 1.093 & 2 & 34.319 \\
3D & 4.48 & 1.137 & 2 & 33.942 \\
\hline
\end{tabular}

Furthermore, the column-level capacitance reveals an even smaller increase in $10 \mathrm{aF}$, resulting in a minor difference in the conversion gain $\left(\Delta C G=0.377 \mu \mathrm{V} / \mathrm{e}^{-}\right)$and the $\mathrm{AC}$ response of the two-layer pixel $\left(\Delta \mathrm{f}_{\mathrm{C}}=0.244 \mathrm{~Hz}\right)$ compared to the single-layer one. The low 3DSI impact on the CG also suggests that noise performance will not be degraded.

The diagram of Figure 11 shows the output voltage of SF versus the number of photogenerated electrons at $\mathrm{SN}$, where it is apparent that there is a constant vertical shift of the response without significant change in its slope (CG remains the same) while varying TG bias. The former can be considered as an offset that can be easily adjusted during the readout process.

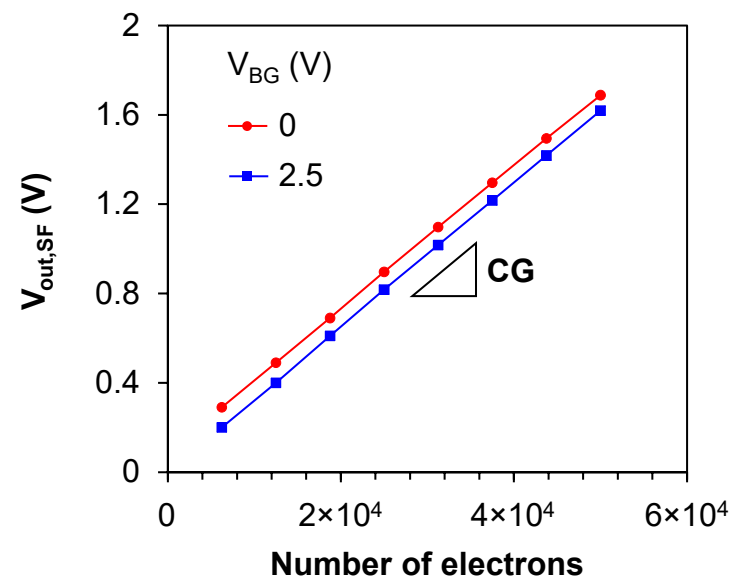

Figure 11. Output voltage of the in-pixel SF transistor versus the number of photo generated electrons at $\mathrm{SN}$ for the voltage bias limits of the bottom tier TG $(0 \mathrm{~V}-2.5 \mathrm{~V})$. The slope provides that $\mathrm{CG}$ was not altered with TG coupling. 
Concerning the rest of pixel performance metrics, they are either related to the photodiode technology and size (similarly to FWC and FF) and, thus, are not affected by 3DSI layering, or directly related to the CG, such as the SNR and DR, which was found almost unchanged by parasitic coupling.

Continuing our analysis, in order to estimate the impact of the inter-tier coupling on the transient response of the top readout circuit, the two scenarios of Figure 12 were employed. The first is considered when SF is placed above a TG of the same pixel (Figure 12a). In that case, charge transfer occurs right at the time in which TG is enabled, as shown in the pixel readout cycle. Thus, sampling processes performed right before and after the charge transfer cannot result in an error.

a. SF above TG of same pixel

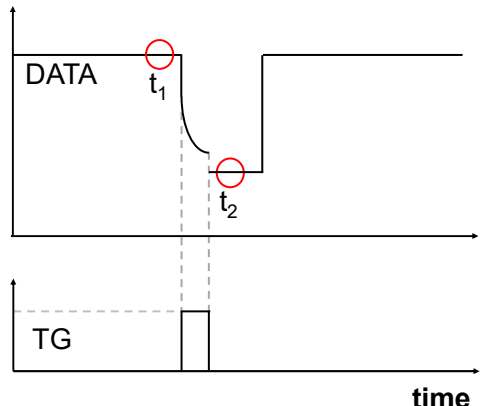

b. SF above TG of adjacent pixel

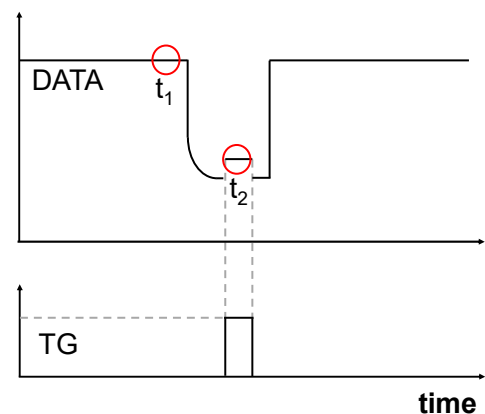

Figure 12. (a) For SF above a TG of the same pixel, TG switches ON during the transfer of $\mathrm{e}^{-}$ from PPD to the SN. Sampling is, thus, performed at $t_{1}$ and $t_{2}$ without readout errors. (b) In the scenario of an SF placed above a TG of an adjacent pixel, sampling can contain erroneous value due to TG coupling.

On the contrary, our second scenario considers an SF placed above the TG of an adjacent pixel (Figure 12b). In this case, TG is not synchronized with the pixel readout; hence, sampling can contain erroneous information. This sampling error $\Delta \mathrm{V}_{\text {out,SF }}$ has been extracted versus the number of the photogenerated electrons at the $\mathrm{SN}$ and for various bias currents of the SF, with the results shown in Figure 13a. In order to evaluate the strength of this error, a comparison was made with the readout error resulting in temperature variations ( $253 \mathrm{~K}-353 \mathrm{~K})$, which is presented in Figure 13b. It is evident that the couplinginduced readout error is significant compared to the temperature-induced one, especially for low light conditions and low bias current.
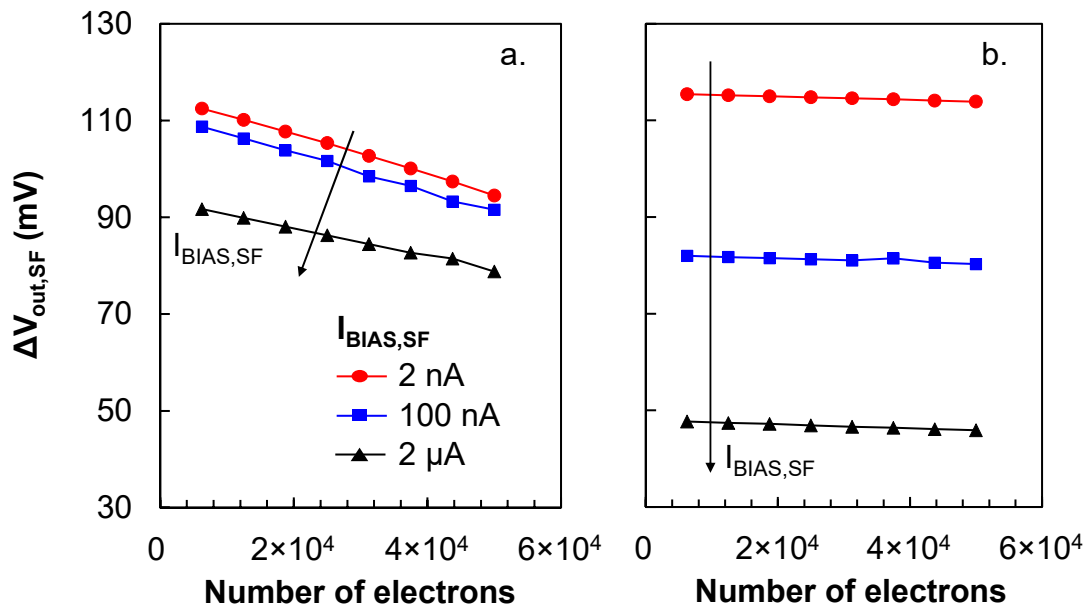

Figure 13. Readout error of the in-pixel SF transistor versus the number of photo generated electrons at SN resulting from (a) SF-TG coupling of Figure $7 \mathrm{~b}$ and (b) sensor temperature variations. 


\subsubsection{Inter-Tier Ground Plane Necessity}

Recent studies [22,23] have demonstrated efficient decoupling solutions between sensitive tiers in 3DSI via the process integration of a conductive layer, i.e., an inter-tier Ground Plane (GP).

Depending on each application's sensitivity and functionality, an inter-tier Ground Plane integration must be considered, taking severely into account process complexity. Nevertheless, in cases where an effective isolation between tiers in 3DSI is required, studies show that the integration of an inter-tier GP made of polysilicon can offer a reduction in vertical static coupling by five orders of magnitude [23].

Concerning the example of the two-layer 3DSI pixel studied in this work, in a typical rolling readout operation, sequential pixel activation implies that there is no probability for a readout error due to TG coupling. Consequently, TG will be enabled outside the readout cycle of this pixel and, thus, does not interfere. Furthermore, the Correlated Double Sampling (CDS) stage that exists commonly after the readout circuit eliminates possible readout errors. Therefore, the direct stacking of the readout tier upon the photodiode area is safe without the necessity for electrical isolation in a 3DSI CIS. However, an intertier GP is mandatory in cases where sensitive blocks are considered to be placed above the photodiode area, such as in-pixel frame memory for which the leakage current is a critical parameter.

\section{Conclusions}

To summarize, we have presented an investigation of coupling-induced effects in a 3DSI PMOS pixel with the aid of TCAD simulations. Our results show that coupling from TG can cause an electrical parameter variation as important as the one induced by a 100 degrees temperature variation. For switch transistors, where the leakage is a critical parameter, this could be a very limiting effect. However, for the in-pixel SF transistor, we demonstrated that the impact of inter-tier electrical coupling on the CG and the AC performance is negligible. Concerning the rest of the indicators presented in Section 2.3, none of them should be affected (especially QE and FWC since they only depend on the photodiode technology). Hence, in addition to the very slight increase in CG, pixel performance should be maintained.

We have further shown that SF-TG coupling may cause a readout error if SF is placed above the TG of a nearby pixel, i.e., in the case that it is not synchronized with the pixel readout. This is not necessarily a limitation for the CIS performance, because if the readout is performed following the rolling shutter scheme, pixel activation is sequential. Therefore, despite strong electrical coupling and high threshold voltage shifts $(\sim 100 \mathrm{mV})$ for top-tier devices, we demonstrated that a sequentially integrated 3D CIS can have an inherent immunity to inter-tier coupling, with zero readout errors.

Author Contributions: Conceptualization, P.S., P.B., G.S. and C.T.; methodology, P.S., A.P., G.S. and C.T.; software, P.S.; validation, P.S. and A.P.; formal analysis, P.S., A.P. and G.S.; investigation, P.S., A.P. and G.S.; resources, P.B.; data curation, P.S.; writing-original draft preparation, P.S. and C.T.; writing-review and editing, A.P., P.B. and G.S.; visualization, P.S.; supervision, A.P., G.S. and C.T.; project administration, P.B.; funding acquisition, P.B., G.S. and C.T. All authors have read and agreed to the published version of the manuscript.

Funding: This work was partially supported by the H2020 3DMUSE European project (Funding number: 780548).

Institutional Review Board Statement: Not applicable.

Informed Consent Statement: Not applicable.

Data Availability Statement: Data will be available upon publication.

Acknowledgments: The authors would like to thank Josep Segura for fruitful discussions.

Conflicts of Interest: The authors declare no conflict of interest. 


\section{References}

1. Arden, W.; Brillouët, M.; Cogez, P.; Graef, M.; Huizing, B.; Mahnkopf, R. “More-than-Moore” White Paper; 2010 ; Version 2. Available online: http://www.itrs2.net/uploads/4/9/7/7/49775221/irc-itrs-mtm-v2_3.pdf (accessed on 2 January 2022).

2. Haruta, T.; Nakajima, T.; Hashizume, J.; Umebayashi, T.; Takahashi, H.; Taniguchi, K.; Kuroda, M.; Sumihiro, H.; Enoki, K.; Yamasaki, T.; et al. A 1/2.3inch 20Mpixel 3-layer stacked CMOS Image Sensor with DRAM. In Proceedings of the Digest of Technical Papers-IEEE International Solid-State Circuits Conference, San Francisco, CA, USA, 5-9 February 2017; Institute of Electrical and Electronics Engineers Inc.: Piscataway, NJ, USA, 2017; Volume 60, pp. 76-77.

3. Theuwissen, A. CMOS image sensors: State-of-the-art and future perspectives. In Proceedings of the ESSDERC 2007-37th European Solid State Device Research Conference, Munich, Germany, 11-13 September 2007; pp. 21-27. [CrossRef]

4. Coudrain, P.; Magnan, P.; Batude, P.; Gagnard, X.; Leyris, C.; Vinet, M.; Castex, A.; Lagahe-Blanchard, C.; Pouydebasque, A.; Cazaux, Y.; et al. Investigation of a sequential three-dimensional process for back-illuminated CMOS image sensors with miniaturized pixels. IEEE Trans. Electron Devices 2009, 56, 2403-2413. [CrossRef]

5. Brunet, L.; Batude, P.; Fenouillet-Beranger, C.; Besombes, P.; Hortemel, L.; Ponthenier, F.; Previtali, B.; Tabone, C.; Royer, A.; Agraffeil, C.; et al. First demonstration of a CMOS over CMOS 3D VLSI CoolCube ${ }^{\mathrm{TM}}$ integration on 300mm wafers. In Proceedings of the 2016 IEEE Symposium on VLSI Technology, Honolulu, HI, USA, 14-16 June 2016; IEEE: Piscataway, NJ, USA, 2016; pp. 1-2.

6. $\quad$ Batude, P.; Brunet, L.; Fenouillet-Beranger, C.; Andrieu, F.; Colinge, J.-P.; Lattard, D.; Vianello, E.; Thuries, S.; Billoint, O.; Vivet, P.; et al. 3D Sequential Integration: Application-driven technological achievements and guidelines. In Proceedings of the 2017 IEEE International Electron Devices Meeting (IEDM), San Francisco, CA, USA, 2-6 December 2017; IEEE: Piscataway, NJ, USA, 2017; pp. 3.1.1-3.1.4.

7. Vivet, P.; Sicard, G.; Millet, L.; Chevobbe, S.; Chehida, K.B.; Angel Cubero, L.; Alegre, M.; Bouvier, M.; Valentian, A.; Lepecq, M.; et al. Advanced 3D Technologies and Architectures for 3D Smart Image Sensors. In Proceedings of the 2019 Design, Automation \& Test in Europe Conference \& Exhibition (DATE), Florence, Italy, 25-29 March 2019; IEEE: Piscataway, NJ, USA, 2019 ; pp. 674-679.

8. Cavalcante, C.; Garros, X.; Batude, P.; Tataridou, A.; Lacord, J.; Casse, M.; Theodorou, C.; Karatsori, T.; Gassilloud, R.; FenouilletBeranger, C; et al. Low temperature high voltage analog devices in a 3D sequential integration. In Proceedings of the 2020 International Symposium on VLSI Technology, Systems and Applications, VLSI-TSA 2020, Hsinchu, Taiwan, 10-13 August 2020.

9. Coudrain, P. Contribution au développement d'une technologie d'intégration tridimensionnelle pour les capteurs d'images CMOS à pixels actifs. Ph.D. Thesis, Institut Supérieur de l'Aéronautique et de l'Espace (ISAE), Toulouse, France, 2009.

10. Weckler, G.P. Operation of p-n Junction Photodetectors in a Photon Flux Integrating Mode. IEEE J. Solid-State Circuits 1967, 2, 65-73. [CrossRef]

11. El Gamal, A.; Eltoukhy, H. CMOS image sensors. IEEE Circuits Devices Mag. 2005, 21, 6-20. [CrossRef]

12. Zhao, C.; Kanicki, J.; Konstantinidis, A.C.; Patel, T. Large area CMOS active pixel sensor x-ray imager for digital breast tomosynthesis: Analysis, modeling, and characterization. Med. Phys. 2015, 42, 6294-6308. [CrossRef] [PubMed]

13. Yadid-Pecht, O.; Ginosar, R.; Diamand, Y.S. A Random Access Photodiode Array for Intelligent Image Capture. IEEE Trans. Electron Devices 1991, 38, 1772-1780. [CrossRef]

14. Guidash, R.M.; Lee, T.H.; Lee, P.P.K.; Sackett, D.H.; Drowley, C.I.; Swenson, M.S.; Arbaugh, L.; Hollstein, R.; Shapiro, F.; Domer, S. $0.6 \mu \mathrm{m}$ CMOS pinned photodiode color imager technology. In Proceedings of the Technical Digest-International Electron Devices Meeting, IEDM, Washington, DC, USA, 10 December 1997; pp. 927-929.

15. Yole Developpement Status of the CMOS Image Sensor Industry 2021. Available online: https://s3.i-micronews.com/uploads/ 2021/08/YINTR21167-Status-of-the-CMOS-Image-Sensor-Industry-2021-Sample.pdf (accessed on 2 January 2022).

16. Kadura, L. New FDSOI-Based Integrated Circuit Architectures Sensitive to Light for Imaging Applications; Grenoble Alpes University: Grenoble, France, 2019.

17. Amara, A.; Rozeau, O. Planar Double-Gate Transistor; Amara, A., Rozeau, O., Eds.; Springer: Dordrecht, The Netherlands, 2009; ISBN 978-1-4020-9327-2.

18. Tsuno, M.; Suga, M.; Tanaka, M.; Shibahara, K.; Miura-Mattausch, M.; Hirose, M. Physically-based threshold voltage determination for MOSFET's of all gate lengths. IEEE Trans. Electron Devices 1999, 46, 1429-1434. [CrossRef]

19. Groeseneken, G.; Colinge, J.P.; Maes, H.E.; Alderman, J.C.; Holt, S. Temperature Dependence of Threshold Voltage in Thin-Film SOI MOSFET's. IEEE Electron Device Lett. 1990, 11, 329-331. [CrossRef]

20. Khanna, V.K. Temperature dependence of electrical characteristics of silicon MOS devices and circuits. In Extreme-Temperature and Harsh-Environment Electronics Physics, Technology and Applications; IOP Publishing: Bristol, UK, 2017.

21. Fowler, B. Single Photon CMOS Imaging Through Noise Minimization; Seitz, P., Theuwissen, A.J., Eds.; Springer Series in Optical Sciences; Springer: Berlin/Heidelberg, Germany, 2011; Volume 160, pp. 159-195; ISBN 978-3-642-18442-0.

22. Vandooren, A.; Wu, Z.; Khaled, A.; Franco, J.; Parvais, B.; Li, W.; Witters, L.; Walke, A.; Peng, L.; Rassoul, N.; et al. Buried metal line compatible with 3D sequential integration for top tier planar devices dynamic Vth tuning and RF shielding applications. Dig. Technol. Pap.-Symp. VLSI Technol. 2019, 2019, T56-T57. [CrossRef]

23. Sideris, P.; Andrieu, F.; Colinge, J.P.; Ghibaudo, G.; Theodorou, C.; Lugo-Alvarez, J.; Batude, P.; Brunet, L.; Acosta-Alba, P.; Kerdiles, S.; et al. Inter-tier Dynamic Coupling and RF Crosstalk in 3D Sequential Integration. Technol. Dig.-Int. Electron Devices Meet. IEDM 2019, 2019, 3.4.1-3.4.4. [CrossRef] 